\title{
Introducing Preprints: A Multidisciplinary Open Access Preprint Platform
}

\author{
Preprints Editorial Office \\ MDPI AG, Klybeckstrasse 64, CH-4057 Basel, Switzerland; E-Mail: info@preprints.org; \\ Tel.: +41-61-683-7734
}

Preprints is a multidisciplinary preprint platform that makes scientific manuscripts from all fields of research immediately available at www.preprints.org. Preprints is a free (not-for-profit) open access service supported by MDPI in Basel, Switzerland.

\section{Posting Preprints in All Research Fields}

Several preprint services for specific subject areas have already been successfully established. The open access e-print project arXiv is a successful preprint service in physics, mathematics, computer science, quantitative biology, quantitative finance and statistics [1]. More recently, the Cold Spring Harbor Laboratory launched bioRxiv, which is dedicated to preprints in the field of biology [2]. PeerJ Preprints focuses on biology, medical and life science in addition to computer sciences [3]. Now, with Preprints, our aim is to provide an open access platform that covers all fields of research. After a screening by the editorial staff of Preprints, submissions are published online immediately and are open for comment by other registered users. Submitted manuscripts should not have been published in a peer-reviewed journal before.

\section{We are Making Research Discoverable, Sharable and Citable}

Open science calls for easy access and large-scale dissemination of scientific content. This means that a preprint service requires optimized search, possibilities to share content in scientific communities, reliable archiving, and the possibility to immediately cite content.

- The advanced search function of Preprints allows for a search by keywords in the article or in the comments, author name, author affiliation, subject areas, disciplines, or date posted.

- $\quad$ Readers can share preprints on social media and bookmark them on external services such as CiteULike, Mendeley, BibSonomy, Reddit, or Delicious.

- Preprints will permanently archive full text documents with specialist services that cater for long-term preservation.

- All posted preprints are immediately citable by their assigned digital object identifier (DOI). Preprints encourages authors to post updates of their working manuscript as research progresses. From the first draft to the final document, every version is independently citable with its unique DOI.

Furthermore, we have implemented the article-level metrics service Altmetric on Preprints to track the number of mentions each preprint receives in online sources, such as the news, social media channels, wikis, forums, etc. [4].

\section{Why Posting on Preprints Matters}

\subsection{Establish Precedence and Receive Feedback}

Authors can establish precedence by posting their research results online before anyone else does; this is important in highly competitive research fields. Sharing early research results also helps 
to solicit feedback from colleagues, which in turn improves the quality of papers. Preprints' public commenting feature makes it easy for registered users to post comments using their real name and affiliation. We want to make sure that all comments are transparent and constructive.

\subsection{Credit Referees' Contributions}

Anonymous peer-review process misses the opportunity to credit the contributions made by referees. To fully credit their work, referee information and review reports will be public and citable on Preprints.

\subsection{Transparent Academic Communication}

All comments and replies on a given preprint are public and made available along with the identity of the commenter. This enables readers to understand who contributed to the final version of a scientific paper, how the improvements took place, and to see all the improved versions of the paper.

\section{Future Directions}

Preprints aims to further develop in the future and connect with a scientific, multidisciplinary journal that publishes papers after an open peer-review process.

\section{More Information}

We invite you to visit Preprints at http://www.preprints.org.

\section{References}

1. arXiv. Available online: http://arxiv.org (accessed on 27 April 2016).

2. bioRxiv. Available online: http://biorxiv.org (accessed on 27 April 2016).

3. PeerJ Preprints. Available online: https://peerj.com/preprints/ (accessed on 27 April 2016).

4. Altmetric. Available online: https://www.altmetric.com (accessed on 27 April 2016).

(C) 2016 by the author; licensee Preprints.org, MDPI, Basel, Switzerland. This article is an open access article distributed under the terms and conditions of the Creative Commons Attribution (CC-BY) license (http://creativecommons.org/licenses/by/4.0/). 